\title{
Effet de la préexposition au soleil et du prétraitement au froid, sur l'évolution des populations de bruches dans les systèmes de stockage traditionnels des graines niébé (Vigna unguiculata(L.) Walp.) au Niger
}

\author{
Nafissatou MAHAMADOU AMADOU* et Ali DOUMMA
}

Faculté des Sciences et Techniques, Université Abdou Moumouni de Niamey, BP 10662 Niamey, Niger.

*Auteur correspondant ; E-mail: nafsat36@gmail.com

\author{
Received: 10-05-2021 \\ Accepted: 16-12-2021 \\ Published: 31122021
}

\section{RESUME}

Le niébé, Vigna unguiculata (L.) Walp., est la principale légumineuse alimentaire cultivée en Afrique de l'Ouest. Cependant la difficulté de conservation post récolte des graines limite sa production et sa disponibilité en toute saison. En effet, plusieurs espèces de Coléoptères bruchinae, dont Callosobruchus maculatus Fab est la plus importante, se développent dans les graines de niébé en causant des pertes substantielles. L'infestation débute dans les champs, et se poursuit dans les stocks où les dégâts peuvent être considérables si aucune mesure de protection n'est prise. Les producteurs de niébé font le plus souvent recours à la lutte chimique, dite classique qui malgré son efficacité a vite montré ses limites (persistance des attaques d'insectes nuisibles) et ses conséquences néfastes. Au cours de cette étude, nous avons évalué les effets de deux méthodes physiques de lutte contre ces ravageurs en vue d'une protection saine des graines de niébé stockées : la préexposition au soleil et le prétraitement au froid. Les résultats obtenus ont montré que pour toutes les méthodes testées, les effectifs d'œufs et d'adultes de bruches ont été relativement faibles comparativement au témoin. Parmi les méthodes testées, le prétraitement des graines au froid pendant au moins une semaine semble être la méthode la plus efficace. Au niveau des lots pré-exposés au soleil, qu'il s'agisse de la méthode locale ou de la méthode améliorée, l'exposition des graines durant 4 semaines réduisent de manière considérable l'évolution des populations des bruches dans les systèmes de stockage. Par ailleurs, ces méthodes n'ont pas affecté le pouvoir germinatif des graines stockées. Pour assurer une bonne production des stocks de niébé contre les bruches, il serait préférable de conserver les graines de niébé pendant au moins une semaine au froid avant de les mettre dans les structures de stockages.

(C) 2021 International Formulae Group. All rights reserved.

Mots clés: Post-récolte, prétraitement au froid, préexposition au soleil, burchinae, niébé, stockage, Niger.

\section{Effect of sun pre-exposure and cold pre-treatment on the evolution of bruchid populations in traditional cowpea (Vigna unguiculata (L.) Walp.) seed storage systems in Niger}

\section{ABSTRACT}

Cowpea, Vigna unguiculata (L.) Walp. is the main food legume grown in West Africa. However, the difficulty of post-harvest conservation of seeds limits its production and availability in all seasons. Indeed, several species of bruchinae beetles, of which Callosobruchus maculatus Fab is the most important, develop in 
cowpea seeds causing substantial losses. The infestation starts in the field, and continues in the stocks where damage can be considerable if no protective measures are taken. Cowpea producers most often resort to chemical control, known as classical control, which despite its effectiveness has quickly shown its limitations (persistence of insect pest attacks) and its harmful consequences. During this study, we evaluated the effects of two physical methods of control against these pests for a healthy protection of stored cowpea seeds: pre-exposure to the sun and pre-treatment with cold. Results showed that for all methods tested, bruchid egg and adult numbers were relatively low compared to the control. Of the methods tested, cold pre-treatment of seeds for at least one week appeared to be the most effective method. In the pre-exposed batches, both the local method and the improved method, exposure of the seeds for 4 weeks significantly reduced the evolution of bruchid populations in the storage systems. In addition, these methods did not affect the germination capacity of the stored seeds. To ensure good production of cowpea stocks against bruchids, it would be preferable to keep cowpea seeds for at least one week in cold storage before putting them in the storage structures.

(C) 2021 International Formulae Group. All rights reserved.

Keywords: Post-harvest, cold pre-treatment, sun pre-exposure, burchinae, cowpea, storage, Niger.

\section{INTRODUCTION}

Bruchidius atrolineatus Pic. et Callosobruchus maculatus Fab. sont les principaux ravageurs du niébé en stockage (Sanon et al., 2005). Ces deux espèces apparaissent dans les cultures de niébé dès la période de floraison et s'y reproduisent lors de la fructification et la maturation des gousses (Kam et Sanon, 2016). Les femelles pondent sur les gousses et les graines, les larves pénètrent dans les graines aux dépens desquelles elles assurent leur développement post embryonnaire. Les larves et les nymphes sont donc déjà présentes lorsque les produits de la récolte sont entreposés dans les greniers. (Amevoin et al., 2006).

Pendant l'entreposage des récoltes du niébé, le taux d'infestation initial par les bruches est généralement inférieur à 5\% (Sanon et al., 2005). Plus tard, les stocks infestés et non protégés convenablement peuvent être complètement détruits au bout de quelques mois de stockage (Amevoin et al., 2006). En outre, les dégâts occasionnés se traduisent par une détérioration de la qualité des graines les rendant impropres à la consommation. Face à la pression parasitaire exercée par ces ravageurs sur les stocks de niébé, différentes méthodes de lutte ont été développées (Doumma, 2012) mais la lutte chimique a très souvent été envisagée (Dabiré et al., 2008 ; Egho, 2011 ; Kayombo et al., 2015). En effet, l'utilisation abusive des insecticides chimiques (parfois prohibés), la défaillance de précaution dans leur manipulation et le non-respect des délais de latence sont autant de facteurs qui entraînent la résistance des insectes nuisibles, l'élimination des ennemis naturels de ceux-ci, des problèmes de santé (aux consommateurs et aux agriculteurs) et la pollution de l'environnement. A ces problèmes s'ajoutent des difficultés d'accès aux marchés (surtout des pays développés), à cause du non-respect des limites maximales de résidus (LMR) de pesticides pour les denrées alimentaires destinées à l'exportation.

Dans cette optique, des efforts importants sont déployés en vue de mettre au point des méthodes alternatives de lutte contre ces bruches. C'est ainsi que des pratiques parfois très anciennes connaissent actuellement un regain d'intérêt et sont testées afin d'offrir une désinsectisation raisonnée qui n'est pas basée sur l'utilisation des pesticides. C'est le cas du traitement au froid notamment dans les pays développés et l'exposition solaire des graines avant leur stockage dont l'utilisation connait de nos jours un intérêt de plus en plus croissant dans la protection des denrées stockées. Ces méthodes offrent l'avantage d'être moins onéreuse et non toxique tout en conservant les qualités physiques, culinaires, nutritives et les autres propriétés organoleptiques de la graine de niébé (Ebayo et Andjorin, 2018). 
La lutte physique est un procédé par lequel l'homme fait recourt à un facteur physique pour détruire le nuisible. Il peut s'agir du traitement des graines par la chaleur, (Maina et Lalé, 2004 ; Allotey et al., 2012) ou par le froid (Loganathan et al., 2011, Maharjan et al., 2017).

De par le monde et depuis des millénaires, le froid et la chaleur ont longtemps été utilisés soit pour désinfecter les produits stockés, soit pour les protéger contre les infestations d'insectes.

Chaque espèce présente une corrélation spécifique entre la température et son développement qui lui est spécifique (Moiroux et al., 2014). Des températures extrêmes provoquent chez les insectes la dénaturation de certaines protéines, l'inactivation d'enzymes et des dérèglements du fonctionnement des membranes plasmiques (Davies et al., 2006). Des valeurs thermiques plus fortes ou plus faibles diminuent le taux d'éclosion des œufs, influencent la durée du développement, aussi bien embryonnaire que larvaire et aboutissent à une mortalité embryonnaire importante. De même, l'exposition des adultes à des températures extrêmes induit une diminution de la longévité (Brodeur et al., 2013). En limitant considérablement le phénomène de thermorégulation, les températures élevées quant à elles provoquent la mort immédiate de l'insecte exposé. Cependant, les études menées jusque-là ont porté pour la plupart des cas sur l'effet de ces méthodes physiques sur les différents stades de développement des ravageurs. Peu d'études ont été consacrées à l'utilisation de ces deux méthodes comme moyens de prévention des dégâts occasionnés par ces ravageurs au cours du stockage.

Il est donc important d'évaluer l'effet de ces deux méthodes physiques de lutte sur les populations des deux espèces de bruches à savoir $C$. maculatus et B. atrolineatus au cours du stockage. Il s'agira particulièrement, pour chacune de ces deux méthodes physiques, d'évaluer l'effet du prétraitement permettant de réduire les dégâts occasionnés par ces ravageurs au cours du stockage post-récolte des graines de niébé.

\section{MATERIEL ET METHODES}

\section{Souche de niébé}

Au cours de cette étude, les graines d'une variété locale de niébé, V. unguiculata (L.) Walp ont été utilisées. Il s'agissait de graines infestées naturellement aux champs qui ont été achetées auprès des producteurs de la commune de Baleyera, localité située à 100 $\mathrm{Km}$ au Nord-Est de Niamey, capitale du Niger. Ces graines contenaient à priori tous les différents stades de développement des bruches. Car après l'infestation la larve de $C$. maculatus pénètre à l'intérieur de la graine de niébé pour boucler son cycle de développement.

\section{Méthodologie expérimentale \\ Prétraitement des graines au froid sur les populations de bruches}

L'expérience a consisté à introduire dans un congélateur réglé à $-6^{\circ} \mathrm{C}$ deux lots de $3 \mathrm{Kg}$ de graines de niébé infestées naturellement.

- Le lot CF1 : Les graines de niébé ont été prétraitées au froid pendant 1semaines, avant d'être stockées au laboratoire dans les conditions ambiantes.

- Le lot CF2 : Les graines de niébé ont été prétraitées au froid pendant 2 semaines, avant d'être stockées au laboratoire dans les conditions ambiantes.

- Un lot T0 constitué de $3 \mathrm{Kg}$ de graines de niébé infestées naturellement et maintenu dans les conditions ambiantes a servi de témoin.

Pour chacun des lots expérimentaux et pour le témoin, l'expérience a été répliquée trois (3) fois.

Préexposition des graines au soleil sur les populations de bruches

Méthode préexposition directe des graines au soleil

C'est une méthode couramment utilisée par les producteurs ruraux qui avant de stocker les graines dans les structures de stockage, les exposent préalablement au soleil pendant une période donnée. Pour simuler cette méthode locale, $3 \mathrm{Kg}$ de graines de niébé infestées naturellement ont été étalées sur une natte confectionnée à base de feuilles sèches de 
palmier doum (Hyphaenae thebaica). Deux lots ont été préparés :

- Le lot ES2 : Les graines de niébé ont été pré-exposées au soleil pendant 2 semaines, avant d'être stockées au laboratoire.

- Le lot ES4 : Les graines de niébé ont été pré-exposées au soleil pendant 4 semaines, avant d'être stockées dans les structures de stockage.

- Un lot T0 constitué de $3 \mathrm{Kg}$ de graines de niébé infestées naturellement et maintenu dans les conditions ambiantes a servi de témoin.

Pour chacun des lots expérimentaux et pour le témoin, l'expérience a été répliquée trois (3) fois.

\section{La méthode améliorée d'exposition au soleil}

La méthode testée au cours de cette étude a été inspirée de celle de Murdock et Shade (1991) Elle a consisté à étaler des graines de niébé infestées sur un plastique noir en polyéthylène $(2 \mathrm{~m} \times 0,9 \mathrm{~m})$ qui était luimême étalé sur une natte qui sert d'isolant. Les graines ont été par la suite recouvertes d'un plastique en polyéthylène transparent $(2 \mathrm{~m} \mathrm{x}$ $1 \mathrm{~m})$. Puis les bords des deux plastiques ont été repliés et maintenus en place par des pierres de manière à assurer une certaine étanchéité au dispositif. Les lots suivants ont été préparés :

- Le lot ESA2 : Les graines de niébé ont été exposées au soleil pendant 2 semaines avant d'être stockées au laboratoire.

- Le lot ESA4 : Les graines de niébé ont été exposées au soleil pendant 4 semaines avant d'être stockées au laboratoire.

- Un lot T0 constitué de $3 \mathrm{Kg}$ de graines de niébé infestées naturellement et maintenu dans les conditions ambiantes sert de témoin.

Pour chacun des lots expérimentaux et pour le témoin, l'expérience a été répliquée trois (3) fois.

\section{Paramètres étudies}

Pour le suivi des populations des deux espèces de bruches, les observations ont été réalisées tous les quinze (15) jours. A chaque date d'observation, les systèmes expérimentaux de stockage ont été d'abord introduits dans un réfrigérateur pendant cinq (05) minutes afin d'immobiliser les insectes et faciliter les manipulations. Ensuite, un échantillon de $250 \mathrm{~g}$ de graines a été prélevé de chaque récipient (Kam, 2020) afin de déterminer :

- le nombre d'adultes vivants et morts des deux espèces de bruches

- le nombre d'œufs des deux espèces de bruches sur les graines ;

Après ces observations, les insectes vivants ont été réintroduits dans les dispositifs de stockage en même temps que les graines échantillonnées.

Les données collectées à partir de ces observations ont permis d'estimer les effectifs d'œufs et d'adultes des bruches au cours de la période stockage.

$\mathrm{Au}$ terme de l'expérience, L'effet de chacune des méthodes testées sur le pouvoir germinatif des graines a été aussi évalué. Pour ce faire, un échantillon de 50 graines a été prélevé dans chaque traitement. Ces graines ont été ensuite ensemencées au laboratoire dans des boites de Pétri contenant du papier filtre humidifiée. Le taux de germination a été estimé en comptant le nombre de graines germées au bout de quatre (4) jours.

\section{Analyse et traitement des données}

A la fin de l'expérience, les moyennes des effectifs obtenues dans les différents lots ont été comparées entre eux par un test de Student Newman Keuls au seuil de probabilité de $5 \%$.

\section{RESULTATS}

\section{Effets des traitements sur l'activité de ponte des bruches}

Quel que soit le traitement considéré, les effectifs d'œufs de $C$. maculatus ont été plus importants que ceux de $B$. atrolineatus (Tableau 1). En outre, on a noté, une variation de l'activité de ponte en fonction des traitements. En effet, les effectifs les plus importants d'œufs ont été observés au niveau du lot témoin, au niveau duquel 9874 œufs ont été dénombrés. Contre 74,3 pour CF1, 85,3 pour CF2, 7417 pour ES2, 5047 pour ES4, 4999,6 pour ESA2 et 4488 pour ESA4.

Au niveau des lots prétraités au froid, les effectifs d'œufs de $C$. maculatus observés au niveau du lot prétraité au froid pendant une 
semaine (CF1) ont été significativement plus importants que ceux observés dans les lots prétraités durant 2 semaines au froid (CF2); Concernant, les lots pré-exposés au soleil, qu'il s'agisse de la méthode locale ou de la méthode améliorée, l'activité de ponte de $C$. maculatus a été significativement plus élevée dans les lots pré-exposés deux semaines au soleil (ES2 et ESA 2) que dans les lots pré-exposés durant 4 semaines (ES4 et ESA4). Pour cette méthode de lutte, les effectifs d'œufs observés dans le lot ESA2 ont été relativement plus faibles que ceux observés dans le lot ES4.

Pour tous les lots expérimentaux, les effectifs d'œufs observés dans les lots CF1 et CF2 étaient plus faibles que ceux observés dans tous les autres lots expérimentaux. En comparant les deux méthodes d'exposition au soleil, il a apparu que les effectifs d'œufs observés ont été plus importants dans les lots exposés directement au soleil que ceux traités avec la méthode améliorée. Concernant $B$. atrolineatus, les effectifs d'œufs déposés ont été relativement faibles au niveau de tous les traitements avec des effectifs ne dépassant pas 22,67 œufs.

\section{Effets des traitements sur les émergences des bruches}

Les émergences de bruches ont été significativement plus importantes dans lot témoin que dans les lots expérimentaux (Tableau 2). En effet, ces effectifs $(7234,16)$ sont 750 fois supérieurs à ceux observés dans les lots prétraités au froid (10 pour CF1 et 9,66 pour $\mathrm{CF} 2$ ), 5 à 10 fois supérieurs à ceux observés respectivement dans les lots ES2 $(1227,66)$ et ES4 $(682)$ et 3 fois supérieur à ceux observés dans les lots ESA2 (2221) et ESA4 (1993,3). Concernant, B. atrolineatus, les effectifs ont été faibles dans tous les traitements.

\section{Effets des traitements sur l'évolution de l'activité de ponte et des émergences des bruches au cours du stockage Evolution de l'activité de ponte}

Les effectifs de $C$. maculatus augmentent significativement dans le lot témoin où un pic est observé au $75^{\text {ème Jours }}$ après Stockage. Par contre, dans les lots traités (ES2, ES4, ESA2, et ESA4), les effectifs de $C$. maculatus, faibles en début de stockage augmentent significativement pour atteindre un pic au $90^{\text {ème }}$ jour de stockage pour finir par chuter par la suite. (Figure 1). Pour les lots CF1 et CF2, l'évolution de l'activité de ponte est restée relativement négligeable durant toute la période de stockage. Pour les autres lots expérimentaux, l'évolution des effectifs d'œufs de C. maculatus a été plus lente dans le lot ES4 que dans les los ES2, ESA2 et ESA4. En effet au niveau de ce lot, les effectifs d'œufs du ravageur sont restés relativement faibles jusqu'aux $75^{\text {ème }}$ Jours après Stockage.

Les effectifs d'œufs de B. atrolineatus relativement importants en début de stockage diminuent considérablement par la suite pour être négligeables à partir du $30^{\text {ème }}$ Jours après Stockage (Figure 2).

\section{Évolution des émergences des bruches}

L'analyse des émergences des adultes de bruches montre que les effectifs d'adultes de C. maculatus sont restés relativement faibles

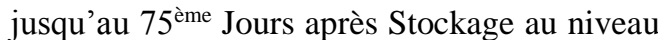
de tous les traitements (Figure 3). A partir de cette date, on note une augmentation importante des effectifs d'adultes de cette espèce dans le lot témoin où un pic est observé

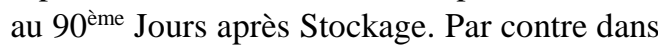
les lots expérimentaux, cette augmentation s'est faite de manière relativement lente jusqu'à la fin de la période de stockage. S'agissant des émergences de $B$. atrolineatus (Figure 4), les effectifs d'adultes de cette espèce, relativement importante en début de stockage dans tous les lots, diminuent considérablement pour devenir négligeable à partir du $30^{\text {ème }}$ Jours après Stockage.

Effet sur le pouvoir germinatif des graines de niébé

Quel que soit le traitement, le taux de germination des graines était supérieur à $94 \%$. En outre aucune différence significative n'a été observée entre les taux de germination enregistré au niveau des différents traitements. (Tableau 3). 
Tableau 1: Effectifs d'œufs de B. atrolineatus et $C$. maculatus dénombrés dans les différents traitements.

\begin{tabular}{lcc}
\hline & $\begin{array}{c}\text { Effectifs d'œufs de Callosobruchus } \\
\text { maculatus }\end{array}$ & $\begin{array}{c}\text { Effectifs d'œufs de Bruchudus } \\
\text { atrolineatus }\end{array}$ \\
\hline Témoin & $9874^{\mathrm{a}}$ & $22,67^{\mathrm{a}}$ \\
\hline CF1 & $74,33^{\mathrm{b}}$ & $21,72^{\mathrm{a}}$ \\
\hline CF2 & $65,33^{\mathrm{c}}$ & $20,56^{\mathrm{a}}$ \\
\hline ES2 & $7417^{\mathrm{d}}$ & $22,43^{\mathrm{a}}$ \\
\hline ES4 & $5047,66^{\mathrm{e}}$ & $20,21^{\mathrm{a}}$ \\
\hline ESA2 & $4993,66^{\mathbf{f}}$ & $22,81^{\mathrm{a}}$ \\
\hline ESA4 & $4488,33^{\mathrm{a}}$ & $19,87^{\mathrm{a}}$ \\
\hline P-value & $<001$ & $<001$ \\
\hline
\end{tabular}

Les valeurs d'une même colonne suivies des différentes lettres alphabétiques présentent de différence significative selon le test de Student Newman Keuls au seuil de probabilité de 5\%.

Tableau 2 : effectifs d'adultes de $B$. atrolineatus et de $C$. maculatus dénombrés dans les différents traitements.

\section{Callosobruchus maculatus}

\begin{tabular}{lcc}
\hline Témoin & $7234,16^{\mathrm{a}}$ & $9,31^{\mathrm{a}}$ \\
\hline CF1 & $10^{\mathbf{b}}$ & $9,12^{\mathrm{a}}$ \\
\hline CF2 & $9,66^{\mathrm{c}}$ & $9,63^{\mathrm{a}}$ \\
\hline ES2 & $1227,66^{\mathrm{d}}$ & $8,47^{\mathrm{a}}$ \\
\hline ES4 & $682^{\mathrm{e}}$ & $9,51^{\mathrm{a}}$ \\
\hline ESA2 & $2221^{\mathrm{f}}$ & $8,03^{\mathrm{a}}$ \\
\hline ESA4 & $1993,33^{\mathrm{g}}$ & $9,1^{\mathrm{a}}$ \\
\hline P-value & $<001$ & $<001$
\end{tabular}

Les valeurs d'une même colonne suivies des différentes lettres alphabétiques présentent de différence significative selon le test de Student Newman Keuls au seuil de probabilité de 5\% 


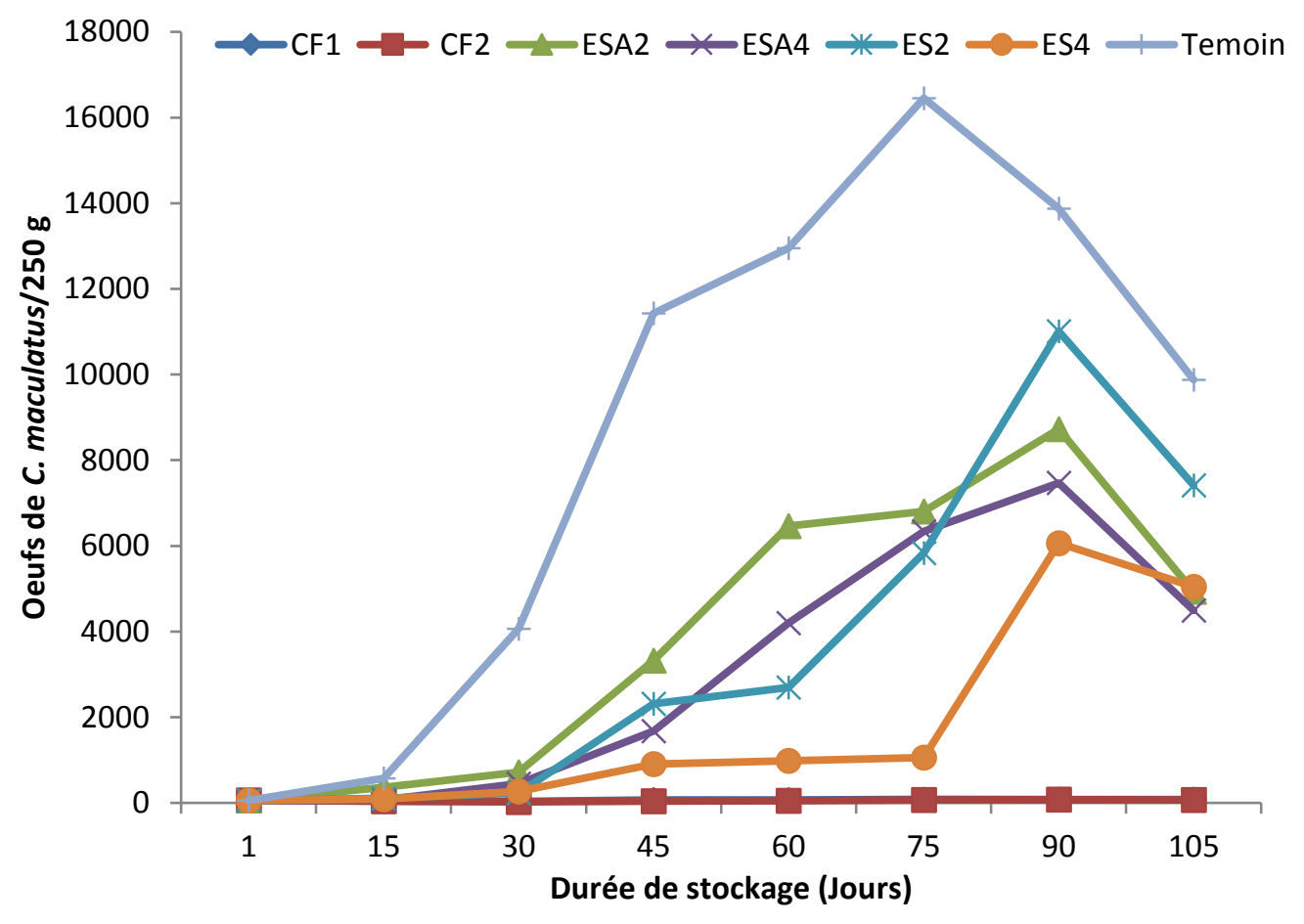

Figure 1: Evolution des effectifs moyens d'œufs de C. maculatus dans les différents traitements.

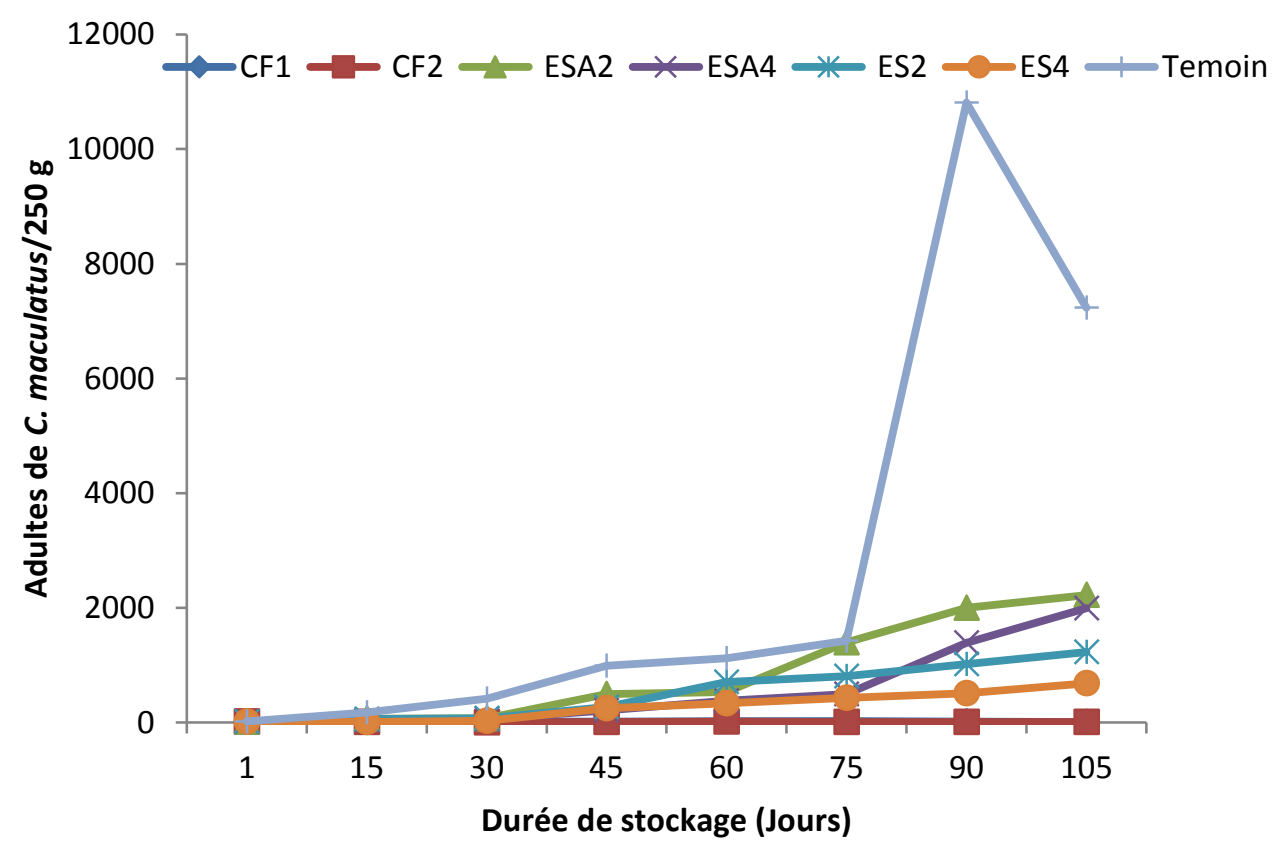

Figure 2: Evolution des effectifs d'adultes de C. maculatus dans les différents traitements. 


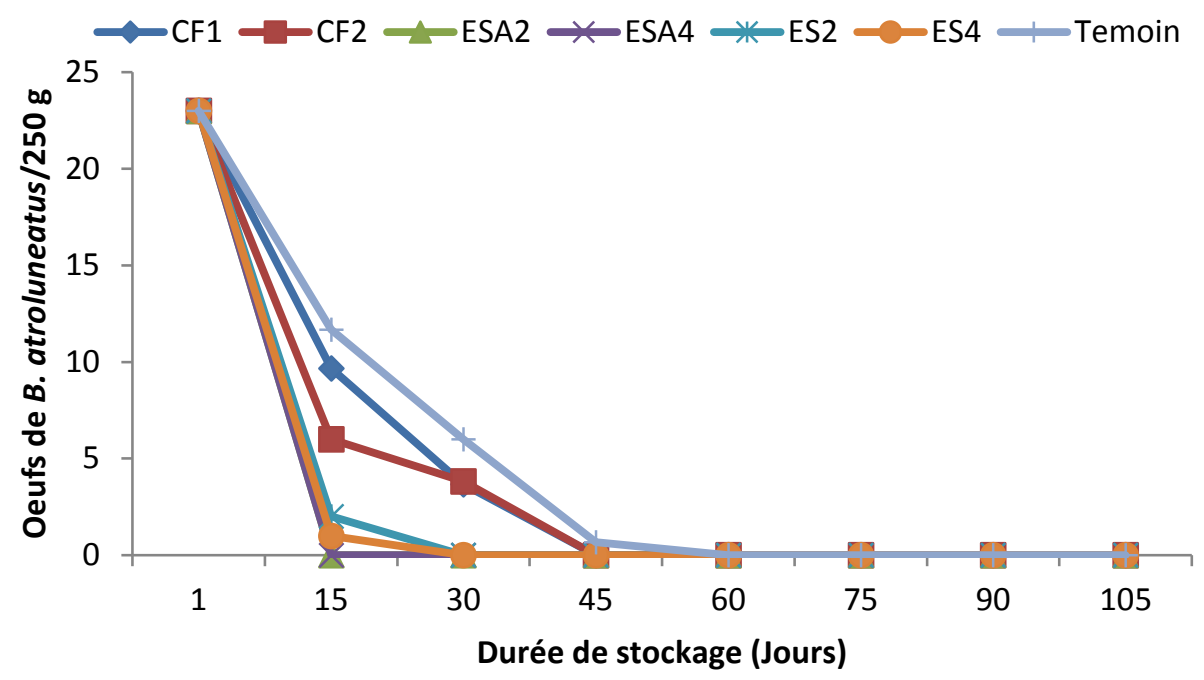

Figure 3: Evolution des effectifs moyens des œufs de B. atrolineatus en fonction des traitements.

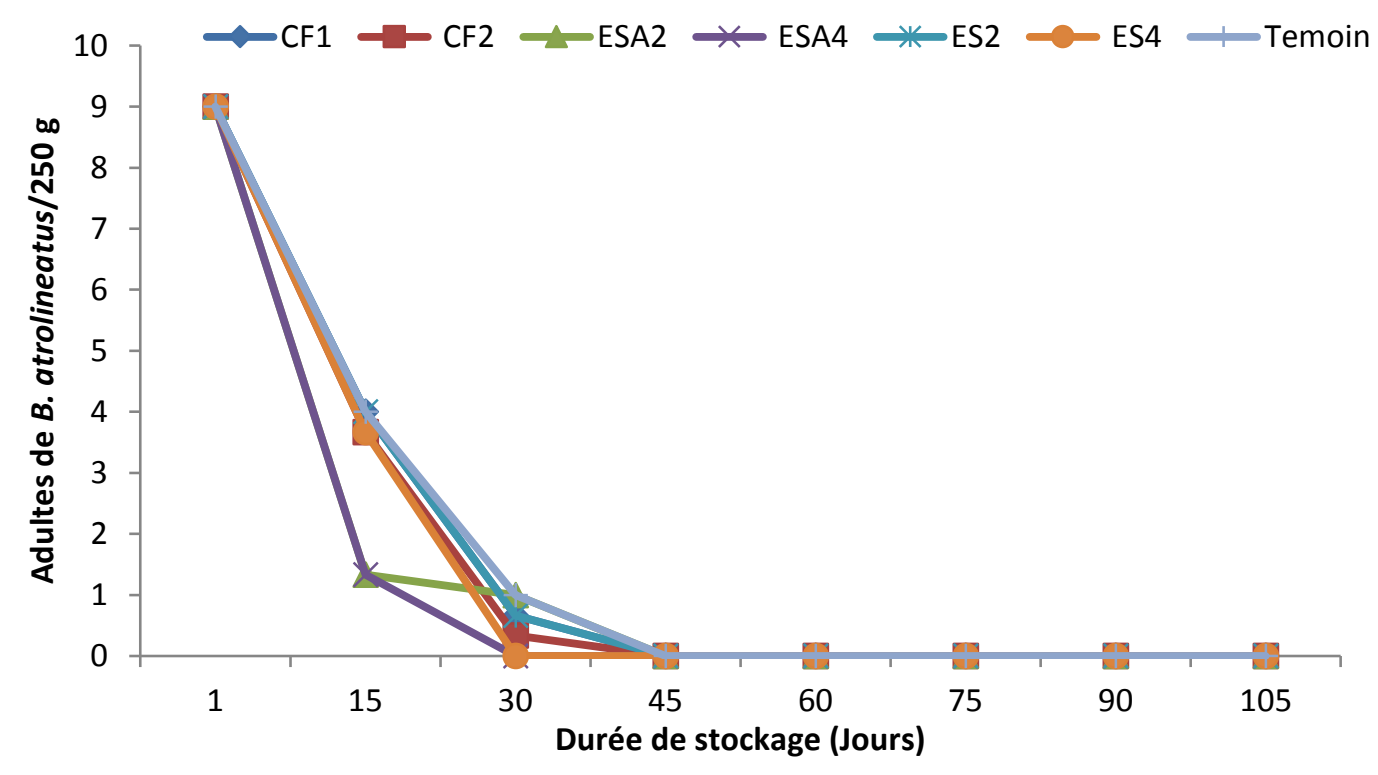

Figure 4: Evolution des effectifs moyens de B. atrolineatus dans les différents traitements.

Tableau 3: Taux de germination observés dans les différents traitements à la fin du stockage.

\begin{tabular}{lccccccc}
\hline & Témoin & CF1 & CF2 & ES2 & ES4 & ESA2 & ESA4 \\
\hline $\begin{array}{l}\text { Graines } \\
\text { Germées }\end{array}$ & $47,50^{\mathbf{a}}$ & $47^{\mathbf{a}}$ & $46,67^{\mathbf{a}}$ & $47^{\mathbf{a}}$ & $47,33^{\mathbf{a}}$ & $48^{\mathbf{a}}$ & $47,33^{\mathbf{a}}$ \\
\hline $\begin{array}{l}\text { Taux de } \\
\text { Germination }\end{array}$ & $95 \%$ & $94 \%$ & $93,34 \%$ & $94 \%$ & $94,66 \%$ & $96 \%$ & $94,66 \%$ \\
\hline
\end{tabular}

Les valeurs d'une même colonne suivies des mêmes lettres alphabétiques ne présentent aucune différence significative. 


\section{DISCUSSION}

Les résultats obtenus au cours de cette étude montrent que quel que soit le traitement les dégâts les plus importants ont été occasionnés par les populations de $C$. maculatus, ces résultats sont différents de ceux observés par Doumma et al. (2006) et par Anna et al. (2015) qui ont montré qu'en stockage, $B$. atrolineatus causent plus de dégâts que de $C$. maculatus. Ces résultats pourraient s'expliquer par le démarrage tardif des activités qui a probablement coïncidé avec la période d'induction de la diapause chez $B$. atrolineatus et de levée de quiescence chez $C$. maculatus. La diapause et la levée de quiescence pourraient influencer les résultats car au cours de la diapause les effectifs de $B$. atrolineatus demeurent faibles, alors que la levée de la quiescence entraine une augmentation des effectifs de $C$. maculatus. En effet, des suivis des populations de $B$. atrolineatus effectués dans des greniers expérimentaux au Niger ont montré que $B$. atrolineatus est peu adaptée aux stocks au niveau desquels il est observé une diminution progressive de ses effectifs dès les premiers mois de stockage. Les adultes de $B$. atrolineatus disparaissent également des stocks à la fin de janvier ou de février (Figure 4), soit 2 à 3 mois après le début de la mise en grenier. Deux à trois générations de cette espèce ont pu se développer dans les stocks. Cette diminution s'explique par l'apparition de la diapause induite chez cette espèce par les conditions thermo et photopériodiques. Ces conditions d'induction de cette diapause sont par contre favorables à la levée de la quiescence chez l'espèce sympatrique $C$. maculatus dont les populations continueront à augmenter dans les stocks. Cependant, il ressort de l'examen des résultats que toutes les méthodes testées influençaient négativement l'activité de ponte et le développement des deux espèces de bruches au cours du stockage. En effet, quel que soit le traitement appliqué, les effectifs d'œufs et d'adultes de C. maculatus qui représentaient l'espèce la plus dominante, ont été plus importants dans le témoin que dans les lots expérimentaux.
Parmi les méthodes de lutte testées, le prétraitement des graines au froid pendant au moins une semaine semble être celle qui limite le plus l'évolution des populations des bruches dans les systèmes de stockage. Selon (Ouafa Ourabiah, 2020), les insectes ravageurs des denrées entreposées cessent de s'alimenter et ne se reproduisent pas à des températures inférieures à $18^{\circ} \mathrm{C}$. L'exposition à des températures plus basses peut tuer les insectes. Ainsi, une exposition de la graine à une température de $-5^{\circ} \mathrm{C}$ pendant 12 semaines permet d'y éliminer les insectes à tous les stades de leur développement. Au niveau des lots pré-exposés au soleil, qu'il s'agisse de la méthode locale ou de la méthode améliorée, l'exposition des graines durant 4 semaines réduisent de manière considérable l'évolution des populations des bruches dans les systèmes de stockage. Toutefois, l'exposition directe des graines au soleil durant 4 semaines semble être celle qui limite le plus l'évolution des populations des bruches dans les systèmes de stockage. Ceci pourrait s'expliquer par le fait que les bruches meurent ou s'évadent dans la nature lors de l'exposition directe des graines au soleil, alors que dans le cas de la méthode améliorée l'étanchéité du dispositif fait que les insectes sont piégés et ne peuvent donc pas s'y échapper. Ces résultats confirment les observations de Guèye et al. (2010) qui montrent que l'insolation permet de faire fuir les insectes grâce à la chaleur et à l'incidence directe des rayons solaires.

Ainsi ces résultats confirment les observations antérieures, qui ont montré que la température est l'un des outils de gestion les plus prometteurs des insectes des denrées stockées (Phillips et Throne, 2010).

Il ressort de l'examen des résultats obtenus au cours de cette étude, que les différents traitements appliqués n'ont eu aucun effet sur le pouvoir germinatif des graines. En effet, quel que soit le traitement considéré, le taux de germination a été supérieur à $94 \%$. 


\section{Conclusion}

Cette étude a été menée dans le but d'évaluer l'efficacité de la préexposition au soleil et du prétraitement au froid, des graines dans la protection des stocks de niébé contre les bruches. L'optimisation de la protection des stocks de niébé contre les bruches peut se faire à travers la lutte physique, et le prétraitement au froid en est un. Les résultats obtenus au cours de cette étude ont montré que toutes les méthodes testées à savoir le prétraitement au froid et la préexposition au soleil des graines limitent considérablement l'évolution des populations des bruches dans les systèmes de stockage de niébé. En effet, pour toutes les méthodes testées, les effectifs d'œufs et d'adultes de bruches ont été relativement faibles comparativement au témoin. Parmi les méthodes testées, le prétraitement des graines au froid pendant au moins une semaine semble être la méthode la plus efficace. Par ailleurs, ces méthodes n'ont pas affecté le pouvoir germinatif des graines stockées. Ainsi, le prétraitement au froid et la préexposition au soleil des graines semblent être des moyens efficaces de protection des stocks de niébé. Toutefois, si l'exposition au soleil peut être à la portée des producteurs ruraux, ceci n'est pas le cas du prétraitement au froid dont la vulgarisation est limitée par la disponibilité et le coût élevé de l'énergie et les moyens financiers pour y faire face. Cependant, cette méthode de prétraitement au froid peut être vulgarisée dans les centres urbains pour la conservation de petits stocks au niveau des ménages.

\section{CONFLIT D'INTERETS}

Aucun conflit d'intérêts n'existe entre les auteurs de ce manuscrit.

\section{CONTRIBUTIONS DES AUTEURS}

MAN est l'auteur qui a conduit la recherche et a rédigé le manuscrit sous la supervision du Professeur AD.

\section{REMERCIEMENTS}

Les auteurs remercient très sincèrement le Centre Régional de l'Agrhymet du Niger et particulièrement le Dr N'baye N'diaye expert formateur et Sama Gagara technicien pour leur contribution importante à la réalisation de ce travail.

\section{REFERENCES}

Adebayo RA, Andjorin OO. 2018. Assessment of Entomocidal Effects of Solar Radiation for the Management of Cowpea Seed Beetle, Callosobruchus maculatus (F.) (Coleoptera: Chrysomelidae) in Stored Cowpea. Global Journal of Science Frontier Research: Agriculture and Veterinary, 18(2): 21-26.

Allotey J, Sefa-Dedeh S, Osei AK, Collison EK. 2012. Comparative study of the effects of steam and solar heat treated cowpea seed on the development and control of Callosobruchus maculatus (F.) (Coleoptera: Bruchidae). African Journal of Food, Agriculture, Nutrition and Development, 3: 6065-6078.

Amevoin K, Glitho IA, Nuto Y, Monge JP. 2006. Dynamique des populations naturelles de bruches et de leurs parasitoïdes nympho-larvophages en situation expérimentale de stockage du niébé en zone guinéenne. Tropicultura, 24(1): 45-50.

ANNA BI, Moumouni DA, Doumma A, Alfari B. 2015. Etude de quelques facteurs influençant la biologie et le comportement de Bruchidius atrolineatus Pic. (Coleoptera-Bruchinae), ravageur du niébe (Vigna unguiculata (L.) Walp en zone sahelienne. Agronomie Africaine, $\mathbf{1}$ : 27-36.

Brodeur J, Boivin G, Bourgeois G, Cloutier C, Doyon J, Grenier P, Gagnon AE. 2013. Impacts des changements climatiques sur le synchronisme entre les ravageurs et leurs ennemis naturels : conséquences sur la lutte biologique en milieu agricole au Québec. Rapport final Ouranos, Québec.

Dabire CLB, Niango MB, Sanon A. 2008. Effects of crushed fresh Cleome viscosa L. (Capparaceae) plants on the cowpea storage pest, Callosobruchus maculatus Fab. (Coleoptera: Bruchidae). International Journal of Pest. Management, 54: 319-326. DOI: https: //doi.org/10.1080/ 09670870802266953

Davies ZG, Wilson RJ, Coles S, Thomas CD. 2006. Changing habitat associations of a 
thermally constrained species, the silverspotted skipper butterfly, in response to climate warming. Journal of Animal Ecology, 75: 247-256. DOI: https://doi.org/10.1111/j.1365-

2656.2006.01044.x

Doumma A, Liman AI, Toudou A, Alzouma I. 2006. Comportement de vingt variétés de niébé de (Vigna unguiculata) vis-à-vis de Bruchidius atrolineatus (Pic) et Callosobruchus maculatus (Coleoptera : Bruchidae). Cahiers Agricultures, 15(2): 187-193.

Doumma A. 2012. Influence de deux alternatives de lutte sur les capacités reproductrices de Callosobruchus maculatus Fab. (Coleoptera-Bruchidae), ravageur du niébé (Vigna unguiculata Walp.). Thèse de Doctorat d'Etat, Université Abdou Moumouni de Niamey, Niamey, 171p.

Egho EO. 2011. Management of major field insect pests and yield of cowpea (Vigna unguiculata (L.) Walp.) under calendar and monitored application of synthetic chemicals in Asaba, southern Nigeria. Am. J. Sci. Ind. Res., 2(4): 592-602. DOI: 10.5251/ajsir.2011.2.4.592.602

Guèye MT, Seck D, Wathelet JP, Lognay G. 2011. Lutte contre les ravageurs des stocks de céréales et de légumineuses au Sénégal et en Afrique occidentale synthèse bibliographique. Biotechnol. Agron. Soc. Environ., 15(1): 183-194.

Kam KW, Sanon A. 2016. Dynamic of bruchid egg-laying in cowpea-based cropping systems: importance for determining an optimal release period of the oophagous parasitoid Uscana lariophaga Steffan (Hymenoptera: Trichogrammatidae). International Journal of Environmental \& Agriculture Research, 5: 22-28. DOI: https://www.academia.edu/25738383

Kayombo MA, Mutombo TJM, Muka MP, Somue MA, Kalambaie BMM. 2015. Effet de la poudre de Tephrosia vogelii dans la conservation des graines de niébé (Vigna unguiculata L. Walp.) en stock contre Callosobruchus maculatus F. à Mbujimayi (RD. Congo). Journal of Animal and Insects Sciences, 25(1): 38273835.

Loganathan M, Jayas DS, Fields PG, White NDG. 2011. Low and high temperatures for the control of cowpea beetle Callosobruchus maculatus (F.) (Colleoptera: Bruchidae) in chickpeas. Journal of Stored Products Research 47: 244-248.

DOI: https://doi.org/10.1016/j.jspr.2011.03.00 5

Maina YT, Lale NES. 2004. Integrated management of Callosobruchus maculatus infesting cowpea seeds in storage using varietal resistance, application of neem seed oil and solar heat. International Journal of Agriculture \& Biology, 6(3): 440-446.

Murdock LL, Shade RE. 1991. Eradication of cowpea weevil (Coleoptera: Bruchidae) in cowpeas by solar heating. American Entomol., 37: 228-231. DOI: https://doi.org/10.1093/ae/37.4.228

Ouafa O. 2020. Etude de l'influence des basses températures sur le développement et la mortalité larvaire de la pyrale des dattes en conditions contrôlées. Mémoire de Master, Université Mohamed Khider de Biskra, Biskra, 34p.

Phillips TW, Throne JE. 2010. Bio-rational Approaches to Managing Stored Product. Annu. Rev. Entomol., 55: 375-397. DOI: https://doi.org/10.1146/annurev.ento.54. 110807.09045

Sanon A, Dabire C, Ouedraogo AP, Huignard J. 2005. Field occurrence of bruchid pests of cowpea and associated parasitoids in a sub humid zone of Burkina Faso: importance on the infestation of two cowpea varieties at harvest. Plant Pathology Journal, 4(1): 14-20. DOI: 10.3923/ppj.2005.14.20 